International Mathematical Forum, 2, 2007, no. 39, 1927 - 1934

\title{
Extremal Polynomials with Varying Measures
}

\author{
Rabah Khaldi \\ Department of Mathematics, Annaba University \\ B.P. 12, 23000 Annaba, Algeria \\ rkhadi@yahoo.fr \\ Fateh Aggoune \\ Guelam University, Faculty of Sciences \\ B.P 401, 24000 Guelma, Algeria \\ faggoune@yahoo.fr
}

\begin{abstract}
We investigate the strong asymptotics for $L_{p}$-extremal polynomials with respect to varying measures on a rectifiable Jordan curve perturbed by a finite Blaschke sequence of point masses outside the curve
\end{abstract}

Mathematics Subject Classification: 42C05, 30E10

Keywords: Extremal Polynomials, Varying measures

\section{Introduction}

Let $\sigma$ be a finite positive Borel measure on a compact set of the complex plane whose support contains an infinite set of points. We denote by $T_{n, 2}(z)=$ $z^{n}+\ldots$, the monic polynomial of degree $n$ orthogonal with respect to the measure $\sigma$. One of the most useful tools in the study of orthogonal polynomials is the fact that they solve the following extremal problem: minimize $L^{2}(\sigma)$ norm for all monic polynonials of degree $n$ i.e.

$$
\left\|T_{n, 2}\right\|_{L^{2}(\sigma)}^{2}:=\min _{Q \in \mathcal{P}_{n-1}}\left\|z^{n}+Q\right\|_{L^{2}(\sigma)}^{2}=m_{n, 2}(\sigma)
$$

where $\mathcal{P}_{n}$ denotes the set of polynomials of degree at most $n$.

This characterization of orthogonal polynomials permits us to define a larger class of polynomials called extremal polynomials that solve the extremal problem: 


$$
\left\|T_{n, p}\right\|_{L^{p}(\sigma)}:=\min _{Q \in \mathcal{P}_{n-1}}\left\|z^{n}+Q\right\|_{L^{p}(\sigma)}^{2}=m_{n, p}(\sigma) .
$$

One of the major areas of research in the study of extremal polynomials is to investigate the strong asymptotics behavior of $T_{n, p}(z)$ as $n \rightarrow \infty$. Other commonly used names are power asymptotic, Szegö asymptotic, or full exterior asymptotic.

The connection of orthogonal polynomials with other branches of mathematics is actually remarkable. We can mention some of them like, moment problems, potential theory, approximation theory, special functions, scattering theory, differential equations, dynamic systems, operator theory, number theory, random matrices, statistics and probability theory, stochastic processes, electrostatics...

There exists an extensive literature on orthogonal polynomials but not enough on extremal polynomials, beginning by Geronimus results in 1952 [15, then the fundamental paper of Widom in 1969 [14],.Kaliaguine in [8], obtained the power asymptotic for extremal polynomials when $E$ is a rectifiable Jordan curve plus a finite set of mass points. Recently, one of the authors studied in $[10,11]$ the case of a measure supported on a rectifiable Jordan curve and circle perturbed by an arbirary Blaschke sequence of point masses.

In the last years extremal polynomials with respect to varying measures have played a major role in several problems of approximation theory and growing attention has been paid to the study of their different types of asymptotics behavior. This is not accedently, they become a powerful tool in solving problems where a fixed measure and orthogonality are involved. We mention that the first person that worked with varying measures was Gonchar [6], then Lopez and Gonchar [7] introduced them for studying general results of convergence of multipoint Padé approximants for Cauchy transformation. Moreover, Ysen and Lopez [4], studied the strong asymptotic of orthogonal polynomials with respect to varying measures in the case of the unit circle and the real line, then they obtain certain relations for the polynomials involved in the contruction of Hermite-Padé approximation of a Nikishin system of functions. We find some resuts concerning the study of the strong asymptotics for extremal polynomials with varying measures in various degree of generality in $[1,3,13]$.

In this work we try to carry over some of the main ideas of [3] for $L_{p^{-}}$ extremal polynomials with respect to varying measures on a rectifiable Jordan curve perturbed by a finite Blaschke sequence of point masses outside the curve, where the situation turns out to be much more difficult. Before setting the main results, let us introduce some notations.

Let $E$ be an analytic Jordan curve in the complex plane, we denote by $B$ the bounded component of the complement of $E$ and let $\varphi$ be the confomal mapping which maps the open unit disc $\mathbf{D}=\{z:|z|<1\}$ onto $B$ such that 
$\varphi(0)=\omega \in B$ and $\varphi^{\prime}(0)>0$. From Caratheodory Theorem the function $\varphi$ an its inverse $\nu=\varphi^{-1}$ have a continuous extention to the unit circle and on $E$, respectively.

Let $\sigma(s)$ be a positive measure on $\left[0, l\left[\right.\right.$, where $l$ is the length of $E$ and $\sigma^{\prime}(s)$ the Radon-Nikodym derivate of $\sigma(s)$ with respect to the Lebesgue measure $|d \xi|$ on $E$, we suppose that it satisfies the Szegö's condition:

$$
\int_{E}\left(\log \sigma^{\prime}(s)\right)\left|\nu^{\prime}(\xi)\right||d \xi|>-\infty
$$

then we see that for the measure $\mu$ defined on the unit circle by

$$
\mu(A)=\sigma\left(\xi^{-1}\left(\nu^{-1}(A)\right)\right)=\sigma\left((\nu \circ \xi)^{-1}(A)\right),
$$

yields

$$
\sigma^{\prime}(s)|d \xi|=\sigma^{\prime}(s)\left|\varphi^{\prime}(\theta)\right| d \theta=\mu^{\prime}(\theta) d \theta
$$

Thus the measure $\mu$ satisfies on the unit circle the usual Szegö's condition:

$$
\int_{0}^{2 \pi} \log \left(\mu^{\prime}(\theta)\right) d \theta>-\infty
$$

Therefore, from the properties of the Szegö function $D_{p}(\mu,$.$) associated with$ the unit circle and the weight function $\mu^{\prime}(\theta)$ (see [12]):

$$
D_{p}(\mu, w)=\exp \left\{\frac{1}{2 p \pi} \int_{0}^{2 \pi} \log \left(\mu^{\prime}(\theta)\right) \frac{1+w e^{-i \theta}}{1-w e^{-i \theta}} d \theta\right\}, w \in \mathbf{D}
$$

we can easily deduce the following properties for the Szegö function $\Delta_{p}(\sigma, z)=$ $D_{p}(\mu, \nu(z))$ associated with the curve $E$ and the weight function $\sigma^{\prime}(\theta)$ :

1) $\Delta_{p}(\sigma,$.$) is analytic on B, \Delta_{p}(\sigma, z) \neq 0$ on $B$, and $\Delta_{p}(\sigma, \omega)>0$.

2) $\left|\nu^{\prime}(\xi)\right|\left|\Delta_{p}(\sigma, \xi)\right|^{p}=\sigma^{\prime}(s)$, almost everywhere on $E$.

\section{Auxiliary results}

We need to introduce the following auxiliary results. But first we set some notations. $H^{p}(\mu)$ is defined as the $L^{p}(\mu)$ closure of the polynomial in $e^{i \theta}$ and $\|f\|_{H^{p}(\mu)}^{p}=\int\left|f\left(e^{i \theta}\right)\right|^{p} d \mu(\theta)$. We denote by $H^{p}=H^{p}(m)$, where $m=\frac{d \theta}{2 \pi}$ is the normalized Lebesgue measure on $\left[0,2 \pi\left[\right.\right.$. Let $K_{p}(\mu, z)$ be the function such that $K_{p}(\mu, z)=\frac{D_{p}(\mu, 0)}{D_{p}(\mu, z)}$ if $z \in\left(S_{a} \cup\{z:|z|<1\}\right.$ and $K_{p}(\mu, z)=0$ for $z \in S_{s}$, 
where $S_{a}$ and $S_{s}$ are a disjoint decomposition of the unite circle such that $\mu^{\prime}$ and $\mu_{s}$ live on these sets respectively. Hereafter, $\mu_{s}$ denotes the singular parts of $\mu$ with respect to the Lebesgue measure. $L_{s}^{2}(\mu)=\left\{f \in L^{2}(\mu): f=0, \mu^{\prime}-a . e\right\}$.

Theorem 1 ([2]). If $\underset{f}{f} \in H^{p}(\mu)$, then there existe unique functions $\widetilde{f}, f_{s}$ such that $f=K_{p} \widetilde{f}+f_{s}, \widetilde{f} \in H^{p}$, and $f_{s} \in L_{s}^{2}(\mu)$.

Now we state Keldysh Theorem:

Theorem 2([9]). Assume that the measure $\mu$ satisfies the Szegő condition and $\left\{f_{n}\right\} \subset H^{p}(\mu), 0<p<\infty$, such that

i) $\lim _{n \rightarrow \infty} \widetilde{f}_{n}(0)=1$;

ii) $\lim _{n \rightarrow \infty}\left\|f_{n}\right\|_{H^{p}(\mu)}=D_{p}(\mu, 0)$

Then

a) $\lim _{n \rightarrow \infty} \widetilde{f}_{n}(z)=1$ holds uniformly on each compact subset of $\mathbf{D}$.

b) $\lim _{n \rightarrow \infty}\left\|f_{n}-K_{p}(\mu, z)\right\|_{H^{p}(\mu)}=0$.

Notice that $H^{p}\left(\sigma^{\prime}\right)$ denotes the space of analytic functions $f$ on. $B$, such that $(f \circ \varphi)\left(\Delta_{p}(\sigma,.) \circ \varphi\right) \in H^{p}$. For $1 \leq p \leq \infty, H^{p}\left(\sigma^{\prime}\right)$ is a Banach space, with the norm:

$$
\|f\|_{H^{p}\left(\sigma^{\prime}\right)}^{p}=\lim _{r \rightarrow 1^{-}} \frac{1}{2 \pi r} \int\left|f\left(\varphi\left(r e^{i \theta}\right)\right) \Delta_{p}\left(\sigma, \varphi\left(r e^{i \theta}\right)\right)\right|^{p} d \theta .
$$

For $0<p<1, H^{p}\left(\sigma^{\prime}\right)$ is a quasi-Banach space.

Lemma 1([8]). If $f \in H^{p}\left(\sigma^{\prime}\right)$ then for every compact set $K \subset B$ there is a constant $C_{K}$ such that:

$$
\sup \{|f(z)|: z \in K\} \leq C_{K}\|f\|_{H^{p}\left(\sigma^{\prime}\right)}^{p} .
$$

\section{Main results}

We conserve the same notations as above. Set

$$
d \alpha_{n}=\sigma_{n}+\gamma=\frac{d \sigma}{\left|Y_{n}\right|^{p}}+\gamma,
$$

where the point measure $\gamma=\sum_{k=1}^{N} A_{k} \delta_{z_{k}}$ is supported on $\left\{z_{k}\right\}_{k=1}^{N} \subset \mathbb{C} \backslash \bar{B}$ with masses $A_{k}>0, k=1, \ldots N$ and $\left\{Y_{n}\right\}_{n=1}^{\infty}$ is a sequence of polynomials such that, for each $n, Y_{n}$ has degree $n$, all its zeros $w_{n, i}, i=1, \ldots, n$ lie in the unbounded component of the complement of the curve $|\Phi(z)|=R>1$, where $\Phi$ is a function which maps the exterior of $E$ onto the exterior of the unite circle $G=\{w:|w|>1\}$.

We will study the asymptotic behavior of polynomials denoted by $T_{n, p}^{N}(z)$ that solve the next extremal problem 


$$
m_{n, p}\left(\alpha_{n}\right)=\min _{P_{n}(\omega)=1}\left\{\frac{1}{2 \pi} \int_{E}\left|\frac{P_{n}(\xi)}{Y_{n}(\xi)}\right|^{p} d \sigma(s)+\sum_{k=1}^{N} A_{k}\left|P_{n}\left(z_{k}\right)\right|^{p}\right\}^{1 / p} .
$$

Denote by $T_{n, p}(z)$ the extremal solution of the following problem

$$
m_{n, p}\left(\sigma_{n}\right)=\min _{P_{n}(\omega)=1}\left\{\frac{1}{2 \pi} \int_{E}\left|\frac{P_{n}(\xi)}{Y_{n}(\xi)}\right|^{p} d \sigma(s)\right\}^{1 / p} .
$$

Theorem 3. Let $0<p<\infty$, then for the extremal value $m_{n, p}\left(\alpha_{n}\right)$ of the problem (3) we have:

$$
\lim _{n \rightarrow \infty} m_{n, p}\left(\alpha_{n}\right)=\Delta_{p}(\sigma, \omega)=D_{p}(\mu, 0)
$$

\section{Proof.}

The extremal property of $T_{n, p}(z)$ implies that

$$
m_{n, p}\left(\sigma_{n}\right) \leq\left\{\frac{1}{2 \pi} \int_{E}\left|\frac{T_{n, p}^{N}(\xi)}{Y_{n}(\xi)}\right|^{p} d \sigma(s)\right\}^{1 / p} \leq m_{n}\left(\alpha_{n}\right)
$$

On the other hand it is proved in [3] that

$$
\lim _{n \rightarrow+\infty} m_{n, p}\left(\alpha_{n}\right)=\Delta_{p}(\sigma, \omega)=D_{p}(\mu, 0)
$$

Using (5) and (6) we get

$$
\liminf _{n \rightarrow+\infty} m_{n, p}\left(\alpha_{n}\right) \geqslant \liminf _{n \rightarrow+\infty} m_{n, p}\left(\sigma_{n}\right)=\Delta_{p}(\sigma, \omega)=D_{p}(\mu, 0) .
$$

Now let $q_{N}(z)$ be the polynomial whose zeros are $z_{1}, \ldots, z_{N}$ and $q_{N}(\alpha)=1$ that is $q_{N}(z)=\prod_{k=1}^{N}\left(z-z_{k}\right) / \prod_{k=1}^{N}\left(\omega-z_{k}\right)$, then the extremal property of $T_{n}^{N}(z)$ implies that

$$
\begin{aligned}
\left(m_{n, p}\left(\alpha_{n}\right)\right)^{p} & \leq \min _{P_{n-N}(\omega)=1}\left\{\frac{1}{2 \pi} \int_{E}\left|\frac{P_{n-N}(\xi)}{Y_{n}(\xi)}\right|^{p}\left|q_{N}(z)\right| d \sigma(s)\right\} \\
& =\frac{1}{2 \pi} \int_{E}\left|\frac{T_{n-N, p}(\xi)}{Y_{n}(\xi)}\right|^{p}\left|q_{N}(z)\right| d \sigma(s)
\end{aligned}
$$


where the polynomials $T_{n-N, p}(z)$ are extremal with respect to the measure $d \eta(s)=q_{N}(z) d \sigma(s)$.

it is prove in [3, th 1.1 p.30] that

$$
\lim _{n \rightarrow \infty}\left\{\frac{1}{2 \pi} \int_{E}\left|\frac{T_{n-N, p}(\xi)}{Y_{n}(\xi)}\right|^{p} d \eta(s)\right\}^{1 / p}=\Delta_{p}(d \eta, \omega) .
$$

From the properties of the Szegö function, we obtains that

$$
\Delta_{p}(d \eta, \omega)=D_{p}(\mu, 0)
$$

So,

$$
\limsup _{l \rightarrow+\infty} m_{n, p}\left(\alpha_{n}\right) \leq D_{p}(\mu, 0)
$$

Inequalities (7) and (8) prove Theorem 3.

Theorem 4. For $0<p<\infty$, the extremal polynomials $T_{n, p}^{N}(z)$ have the following asymptotics behavior $(n \rightarrow \infty)$ :

(i) $\lim \left\|\frac{T_{n, p}^{N}}{Y_{n}}-\psi^{\infty}\right\|_{H^{2}\left(\sigma^{\prime}\right)}=0$

(ii) $T_{n, p}^{N}(z)=\left[\psi^{\infty}(z)+\varepsilon_{n}(z)\right], \varepsilon_{n}(z) \rightarrow 0$ uniformly on the compact subsets of $B$.

Where the function $\psi^{\infty}(z)=\frac{\Delta_{p}(\sigma, \omega)}{\Delta_{p}(\sigma, z)}$

\section{Proof.}

Puting $h_{n}(w)=\frac{T_{n, p}^{N}(\varphi(w)) D_{p}(\mu, w)}{Y_{n}(\varphi(w)) D_{p}(\mu, 0)}=\frac{T_{n, p}^{N}(z) \Delta_{p}(\sigma, z)}{Y_{n}(z) \Delta_{p}(\sigma, \omega)}$, with $z=\varphi(w)$. Since the sequence of functions $\left\{h_{n}\right\}$ satisfy the hypothesis of Theorem 2 , that is

(i) $h_{n}(0)=\frac{T_{n, p}^{N}(\omega) D_{p}(\mu, 0)}{Y_{n}(\omega) D_{p}(\mu, 0)}=1$,

and from Theorem 3 we obtain

(ii) $\lim _{n \rightarrow \infty}\left\|\frac{T_{n, p}^{N}}{Y_{n}}\right\|_{H^{2}\left(\sigma^{\prime}\right)}=\lim _{n \rightarrow \infty} m_{n, p}\left(\alpha_{n}\right)=D_{p}(\mu, 0)$.

Then it yields

$$
\lim _{n \rightarrow \infty}\left\|\frac{T_{n, p}^{N}}{Y_{n}}-\frac{D_{p}(\mu, 0)}{D_{p}\left(\mu, e^{i \theta}\right)}\right\|_{H^{2}\left(\sigma^{\prime}\right)}=0
$$

and

$$
\lim _{n \rightarrow \infty} h_{n}(w)=1
$$


uniformly on each compact subset of $\mathbf{D}$.

From (9) and the fact that $\psi^{\infty}(\xi)=\frac{\Delta_{p}(\sigma, \omega)}{\Delta_{p}(\sigma, \xi)}=\frac{D_{p}(\mu, 0)}{D_{p}\left(\mu, e^{i \theta}\right)}, \xi=\varphi\left(e^{i \theta}\right)$ we get the affirmation (i) of the Theorem.

Now we will present two proofs for the affirmation (ii) of the Theorem.

First proof of (ii): Using (10) and the expression of $h_{n}(w)$ we obtain

$$
\lim _{n \rightarrow \infty} \frac{T_{n, p}^{N}(z)}{Y_{n}(z)}=\frac{\Delta_{p}(\sigma, \omega)}{\Delta_{p}(\sigma, z)}
$$

uniformly on each compact subset of $\mathbf{B}$.

Second proof of (ii): By applying Lemma 1 for the function $\frac{T_{n, p}^{N}}{Y_{n}}-\psi^{\infty}$ which belongs to $H^{2}\left(\sigma^{\prime}\right)$, then for all compact $K \subset B$, we have

$$
\sup _{z \in K}\left|\frac{T_{n, p}(z)}{Y_{n}(z)}-\psi^{\infty}(z)\right| \leq C_{K}\left\|\frac{T_{n, p}^{N}}{Y_{n}}-\psi^{\infty}\right\|_{H^{2}\left(\sigma^{\prime}\right)}^{2} \underset{n \rightarrow \infty}{\rightarrow} 0 .
$$

\section{References}

[1] M. P. Alfaro, M. Bello Hernandez, J. M. Montaner, J. L. Varona, Some asymptotic properties for orthogonal polynomials with respect to varying measures. J. Approx. Theory, 135 (2005), 22-34.

[2] M. Bello Hernandez, F. Marcellan, J. Minguez Ceniceros, Pseudo-uniform convexity in $H^{p}$ and somme extremal problems on Sobolev spaces, Complex Variables Theory and Application, 48 (2003), 429-440.

[3] M. Bello Hernandez, J. Minguez Ceniceros, Asymptotics for extremal polynomials with varying measures, Electronic Transactions on Numerical Analysis, 19 (2005). 29-36.

[4] B.de la Calle Ysern, G. Lopez Lagamasino, Strong asymptotics of orthogonal polynomials with varying measures and Hermite-Padé approximants, Journ.of Comput. appl Math. 99 (1998) 91-103.

[5] L. Geronimus, On some extremal problems in $L_{\sigma}^{(p)}$ spaces, Math. Sbornik 31(1952), 3-23.

[6] A. A. Gonchar, On the convergence of Pade approximants for some classes of meromorphic functions, Mat. Sb. 97(139) (1975), 607-629. 
[7] A. A. Gonchar and G. Lopez, On Markov's theorem for multi-point Pade approximations, Mat. Sb. 105(147) (1978), 512-524.

[8] V.A., Kaliaguine, On asymptotics of $L_{p}$ extremal polynomials on a complex curve $(0<p<\infty)$, J. Approx. Theory, 74 (1993), 226-236.

[9] M. V. Keldysh, Selected papers, Academic press. Moscow, 1985. [in Russian]

[10] R. Khaldi, Strong asymptotics for $L_{p}$ extremal polynomials off a complex curve, J. Appl. Math. 2004 (2004), no 5, 371-378.

[11] R. Khaldi, Strong asymptotics of $L_{p}$-extremal polynomials off the unit circle. Demonstratio Math. Vol. 38, No.3, (2005), 623-632.

[12] G. Szegő, Orthogonal plynomials, 4th ed. Amer. Math. Soc. Colloquium Publ. Vol 23. Amer. Math. Soc. Providence, RI, 1975.

[13] V. Totik, Weighted Approximation with Varying Weight, Lecture Notes in Math.,Vol. 1569, Springer-Verlag, Berlin, 1994.

[14] H. Widom, Extremal polynomials associated with a system of curves in the complex plane, Adv. in Math. 3 (1969), 127-232.

Received: November 9, 2006 